\title{
The correlation between the STOP-Bang score and oxygen saturation during spinal anesthesia with dexmedetomidine sedation
}

\section{Minsu Yun', Jiwook Kim', Sungwon Ryu', Seo Han', and Yusom Shin ${ }^{2}$}

'Department of Anesthesiology and Pain Medicine, Kosin University Gospel Hospital, Kosin University College of Medicine, ${ }^{2}$ Department of Anesthesiology and Pain Medicine, Busan Central Hospital, Busan, Korea

\begin{abstract}
Background: The STOP-Bang questionnaire is a simple screening tool with high sensitivity for the detection of severe obstructive sleep apnea. Predicting airway obstruction would allow the safe management of sedative patients to prevent intraoperative hypoxia. This study was designed to check the correlation between the STOP-Bang score and oxygen saturation $\left(\mathrm{SpO}_{2}\right)$ during sedation and confirm the availability of the STOP-Bang questionnaire as a preoperative exam for predicting the incidence of hypoxia in sedative patient management.
\end{abstract}

Methods: This study included 56 patients who received spinal anesthesia. The pre-anesthesia evaluation was conducted using the STOP-Bang questionnaire. The patients were under spinal anesthesia with an average block level of T10. Dexmedetomidine was infused with a loading dose of $1 \mu \mathrm{g} / \mathrm{kg}$ over $10 \mathrm{~min}$ and a maintenance dose of $0.5 \mu \mathrm{g} / \mathrm{kg} / \mathrm{h}$ until the end of the procedure. The $\mathrm{SpO}_{2}$ of the patients was recorded every $5 \mathrm{~min}$.

Results: The STOP-Bang score was negatively correlated with the lowest $\mathrm{SpO}_{2}$ (coefficient $=$ $-0.774,95 \%$ confidence interval $[\mathrm{Cl}]:-0.855$ to -0.649 , standard error $[\mathrm{SE}]=0.054, \mathrm{P}<$ 0.001). The item of "observed apnea" was the most correlated one with hypoxic events (odds ratio $=6.00,95 \% \mathrm{Cl}: 1.086$ to 33.145 ).

Conclusions: The STOP-Bang score was significantly correlated with the lowest $\mathrm{SpO}_{2}$ during spinal anesthesia, which enabled the prediction of meaningful hypoxia before it occurred in the sedated patients.

Keywords: Dexmedetomidine; Hypoxia; Obstructive sleep apnea; Sedation.

\section{INTRODUCTION}

For regional anesthesia, sedation is widely used for patient comfort and is required in several places in the hospital as well as in the operating room [1]. Dexmedetomidine has a lesser effect on the respiratory system than other sedative drugs such as propofol or benzodiazepines, especially for short-term sedation such as for drug-induced sleep endos- copy [2,3]. However, studies have shown that central sleep apnea does not occur, but obstructive sleep apnea (OSA) may worsen [4]. Of the complications related to sedation, airway obstruction can cause hypoventilation and hypoxia [5]. If hypoxia persists during surgery, stable sedation will be impossible, and the patient may wake up during the procedure, which may cause an interruption or necessitate unintended discontinuation [6].

This is an Open Access article distributed under the terms of the Creative Commons Attribution Non-Commercial License (http://creativecommons.org/licenses/by-nc/4.0) which permits unrestricted non-commercial use, distribution, and reproduction in any medium, provided the original work is properly cited.

Copyright (C) the Korean Society of Anesthesiologists, 2021 
In this regard, OSA is the most common sleep disorder, mainly due to upper respiratory tract obstruction with a prevalence of $10 \%$ in men and $3 \%$ in women aged between 30 and 49 years, and approximately $17 \%$ of men and $9 \%$ of women aged between 50 and 70 years. The incidence of hypoxemia in patients at risk for OSA is high [7]. Likewise, the incidence of difficult mask ventilation or difficult intubation is high [8]. Polysomnography, the standard method for diagnosing OSA, is not time- and cost-effective to apply to all patients for preoperative risk evaluation of OSA.

The STOP-Bang questionnaire uses only eight items to calculate the risk of OSA. The eight items include the patient's body mass index (BMI, gender, age, neck circumference, hypertension under treatment, daily drowsiness, snoring, and apnea during sleep $[9,10]$. It does not require such a long time, and patients feel more comfortable with it. Although it seems simple compared with other screening tools, its sensitivity is sufficiently high to be reliable in clinical fields [11]. Therefore, the STOP-Bang questionnaire, which has the advantages of simple questions and high sensitivity over other OSA screening tools [12,13], was used for this study to identify patients at high-risk of hypoxemia before sedation.

The authors hypothesized that the STOP-Bang score would be correlated with the incidence of hypoxic events during sedation in patients undergoing spinal anesthesia. The STOP-Bang score can be used to predict the degree of desaturation in sedated patients.

The primary outcome of the study was to check the correlation between the STOP-Bang score and oxygen saturation during dexmedetomidine sedation. Additional attempts were made to determine the cut-off value of the STOP-Bang score that can predict hypoxia during sedation and the items with the highest diagnostic values.

\section{MATERIALS AND METHODS}

\section{Study sample}

This study was approved by the Institutional Review Board (no. KUGH 2019-11-032). Preoperative screening of orthopedic and urological patients who underwent sedation with dexmedetomidine after spinal anesthesia was performed. The exclusion criteria were as follows: American Society of Anesthesiologists physical status class of $\geq 3$, lateral decubitus or prone positioning during surgery, problems of the upper respiratory tract, history of severe asthma or chronic obstructive lung disease, refusal of intraoperative sedation, or sensory block higher than T5. Patients with American Society of Anesthesiologists physical status class 3 due to a high BMI value of $>40 \mathrm{~kg} / \mathrm{m}^{2}$ without any other underlying diseases were included for consideration of the purpose of the study. Written informed consent was obtained from all the patients.

The STOP-Bang score of the patient was confirmed through pre-anesthesia evaluation. The STOP-Bang questionnaire consists of four questions and four clinical characteristics. The STOP-Bang score ranged from 0 to 8 points based on eight items (Supplementary Figs. 1, 2; S = Snoring; $\mathrm{T}=$ Tiredness $\mathrm{O}=$ Observed apnea; $\mathrm{P}=$ Being treated for high blood pressure; $\mathrm{B}=\mathrm{BMI}$ over $35 \mathrm{~kg} / \mathrm{m}^{2} ; \mathrm{A}$ = age over 50 years; $\mathrm{N}=$ neck circumference over $43 \mathrm{~cm}$ in males and 41 $\mathrm{cm}$ in females; $\mathrm{G}=$ gender of males). Patients were classified based on the STOP-Bang questionnaire scores into three groups: the high-risk, intermediate-risk, and low-risk of OSA groups (low-risk group, 0-2 points; intermediate-risk group, 3-4 points; high-risk group, 5-8 points or 2-4 points of STOP questions with at least one of $\mathrm{B}, \mathrm{N}$, and $\mathrm{G}$ ). The official STOPBang questionnaire format was used in the design of the study; however, to be considerate of patients, an official Korean translation version was used when surveying the patients. Both forms were provided at www.STOPBANG.ca by Chung et al. [13].

\section{Spinal anesthesia and patient sedation}

The patients were placed in the right or left lateral position for spinal anesthesia while monitoring their noninvasive blood pressure, electrocardiogram, and pulse saturation. After disinfection of the skin with povidone-iodine solution, a 25-gauge spinal needle was used in a median approach between the 3rd and 4th or the 4th and 5th lumbar spinous process while checking the free flow of cerebrospinal fluid, and a heavy bupivacaine 10-12 mg was injected. Immediately after the removal of the spinal needle, the patient was placed in the supine position and waited for approximately 10 minutes to check the sensory block height using the pinprick test. Dexmedetomidine was used for patient sedation during the surgery with a loading dose of 1 $\mu \mathrm{g} / \mathrm{kg}$ for $10 \mathrm{~min}$ followed by a maintenance dose at a rate not exceeding $0.5 \mu \mathrm{g} / \mathrm{kg} / \mathrm{h}$ until the end of the surgery. During dexmedetomidine sedation, vital signs, including $\mathrm{SpO}_{2}$, were measured and recorded at 5-min intervals. End-tidal capnography was used to confirm the apnea 
during sedation. The oxygen mask was applied immediately to the patient when the $\mathrm{SpO}_{2}$ during sedation decreased to $92 \%$ and lasted more than $30 \mathrm{~s}$ or rapidly decreased to $90 \%$ or less. In such cases, maintenance dose adjustment and adequate stimulation to wake up the patient were provided, if necessary. Rescue steps, such as jaw thrust or manual ventilation, were planned as needed.

\section{Statistical analysis}

According to a previous study by Oshita et al. [14] and the pilot data from our center, we assumed that the correlation between the STOP-Bang score and the lowest $\mathrm{SpO}_{2}$ was 0.4 in calculating sample size. Assuming an attrition rate of $10 \%$, a sample size of 51 patients were determined to be an adequate sample size to aim for $80 \%$ power and $5 \%$ type- 1 error.

All statistical analyses were performed using IBM SPSS version 20.0 (IBM Co., USA). The correlation between the lowest $\mathrm{SpO}_{2}$ and the STOP-Bang scores, age, and BMI were analyzed using simple linear regression and multiple linear regression analysis. The receiver operating characteristic (ROC) curve and the area under the curve (AUC) values were calculated to determine the diagnostic usefulness of hypoxic events and the cut-off value of the STOP-Bang score, age, and BMI. The Mann-Whitney $U$ test and the chisquare test were used to compare the age and BMI of the hypoxic and non-hypoxic groups. The correlations between the individual items of the STOP-Bang questionnaire and the hypoxic events were also analyzed with odds ratios and 95\% confidence intervals (CI). Two-sided P values of $<0.05$ represented statistical significance.

\section{RESULTS}

From February 2020 to May 2020, 60 patients were enrolled in the present study. Four patients dropped out due to conversion from spinal anesthesia to general anesthesia (Fig. 1). The patient characteristics, level of spinal block, sedation time, and the STOP-Bang questionnaire composition ratio are summarized in Table 1.

A negative correlation (coefficient $=-0.774,95 \%$ CI: -0.855 to -0.649 , standard error $[\mathrm{SE}]=0.054, \mathrm{P}<0.001$ ) between the STOP-Bang score and the lowest $\mathrm{SpO}_{2}$ value was observed (Table 2). In addition to the STOP-Bang score, age and BMI, which are expected to be associated with hypoxia during sedation, were also evaluated using Spearman cor-

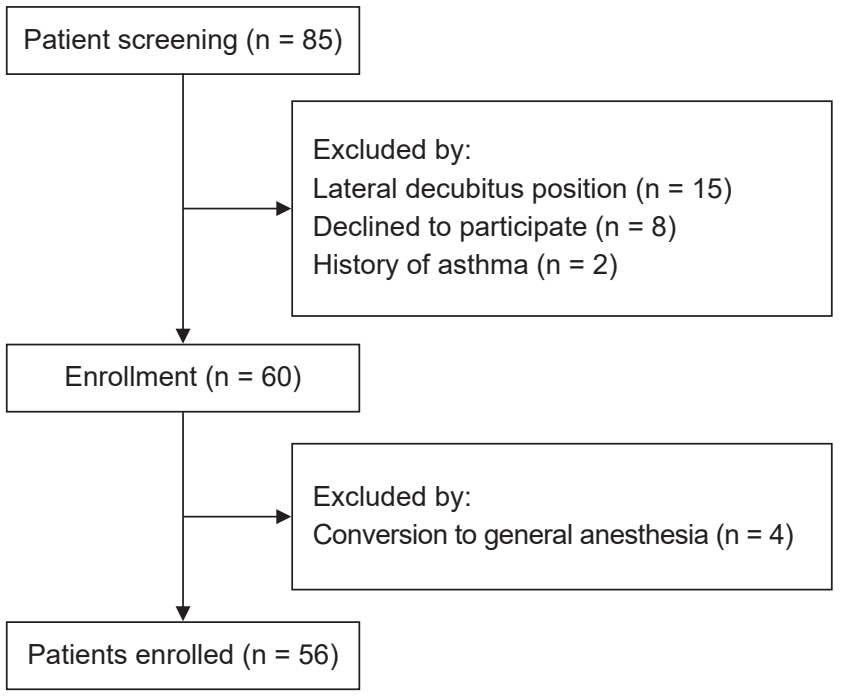

Fig. 1. Flowchart for patient enrollment.

Table 1. Demographic Characteristics of Participants $(n=56)$

\begin{tabular}{|c|c|}
\hline Factor & Value \\
\hline Age (yr) & $64.0 \pm 14.8$ \\
\hline \multicolumn{2}{|l|}{ Gender } \\
\hline Male & $34(60.7)$ \\
\hline Female & $22(39.3)$ \\
\hline Height (cm) & $163.0 \pm 9.8$ \\
\hline Weight (kg) & $62.5 \pm 12.5$ \\
\hline Body mass index $\left(\mathrm{kg} / \mathrm{m}^{2}\right)$ & $24.0 \pm 4.1$ \\
\hline \multicolumn{2}{|l|}{ STOP-Bang score } \\
\hline 0 & $0(0.0)$ \\
\hline 1 & $8(14.3)$ \\
\hline 2 & $14(25.0)$ \\
\hline 3 & $19(33.9)$ \\
\hline 4 & $9(16.1)$ \\
\hline 5 & $6(10.7)$ \\
\hline 6 & $0(0.0)$ \\
\hline 7 & $0(0.0)$ \\
\hline 8 & $0(0.0)$ \\
\hline \multicolumn{2}{|l|}{ STOP-Bang score } \\
\hline Snoring & $25(44.6)$ \\
\hline Tired & $4(7.1)$ \\
\hline Observed & $21(37.5)$ \\
\hline Pressure & $27(48.2)$ \\
\hline Gender & $34(60.7)$ \\
\hline Age $>50$ & $47(83.9)$ \\
\hline $\mathrm{BMI}>35$ & $0(0)$ \\
\hline Neck size & $1(1.8)$ \\
\hline The level of spinal block & $\mathrm{T} 10 \pm 2$ \\
\hline Sedation time (min) & $60.0 \pm 49.1$ \\
\hline
\end{tabular}

Values are expressed as mean \pm SD or number (\%). 
relation analysis. Compared with the STOP-Bang score, BMI was weakly correlated with the lowest $\mathrm{SpO}_{2}$ (coefficient $=$ $-0.288,95 \%$ CI: -0.518 to $-0.013, \mathrm{SE}=0.142, \mathrm{P}=0.031$ ) and age had no correlation with the lowest $\mathrm{SpO}_{2}$ (coefficients = $-0.201,95 \%$ CI: -0.418 to $0.032, \mathrm{SE}=0.114, \mathrm{P}=0.137)$. Scatter plots of the lowest oxygen saturation during sedation and the STOP-Bang score, age, and BMI are shown in Fig. 2.

The ROC analysis of the STOP-Bang score, age, and BMI was performed to determine their usefulness in predicting hypoxic events during sedation. Unlike age and BMI, only the STOP-Bang score was statistically significant (AUC = 0.943, 95\% CI: 0.884 to $1.000, \mathrm{P}<0.001$ ) as a diagnostic tool for hypoxia. The cut-off value of the STOP-Bang score was 4 .

Among 8 items of the STOP-Bang questionnaire, 6 items of STOP were analyzed using odds ratio, and the item of "observed apnea" turned out to be the most correlated one with hypoxic events (Table 3). None of the patients had a BMI of $>35 \mathrm{~kg} / \mathrm{m}^{2}$. Only one patient was included in the neck cir- cumference section. Therefore, a statistical analysis of both items was not possible.

Oxygen masks were immediately applied to eight patients due to intraoperative $\mathrm{SpO}_{2}$ of $<92 \%$ lasting for more than $30 \mathrm{~s}$ or a sudden decrease in saturation to less than $90 \%$ (Table 4). In addition, three patients showed mild bradycardia and were administered glycopyrrolate. After glycopyrrolate injection and a reduction in the dexmedetomidine maintenance dose, the heart rate recovered.

\section{DISCUSSION}

Respiratory depression is a common problem associated with sedation. Dexmedetomidine, a selective $\alpha_{2}$ adrenergic receptor agonist, has sedative and analgesic properties. Its sedation is similar to that of natural sleep; therefore, it is known to cause less respiratory depression [15]. However, the advantage of being similar to natural sleep implies that
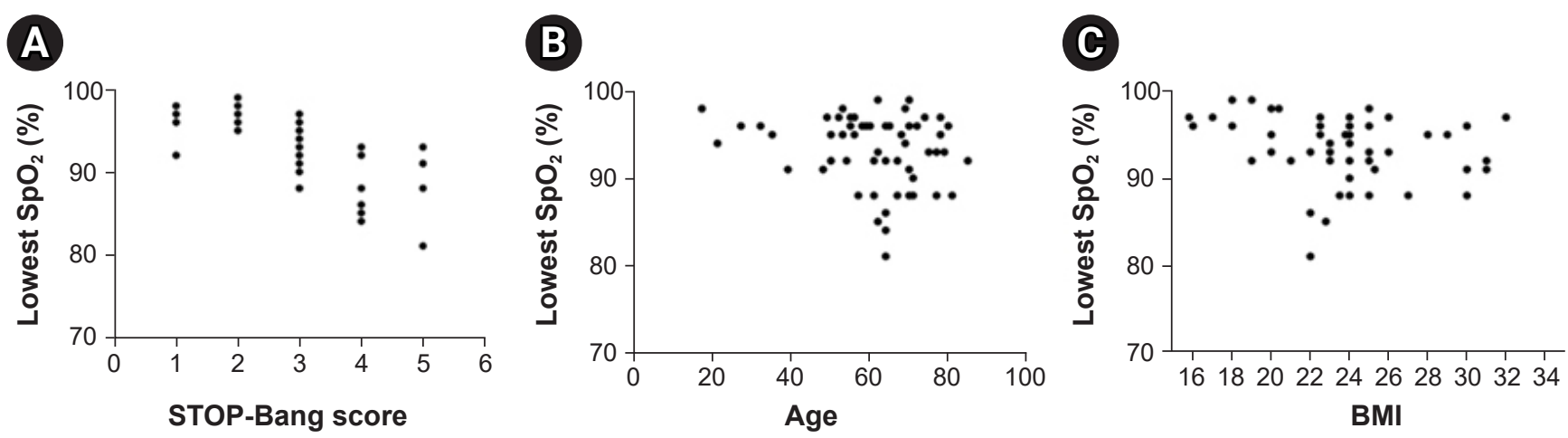

Fig. 2. Scatter plots of lowest oxygen saturation during sedation and various patient factors. The results are shown for (A) STOP-Bang score (B) Age (C) Body mass index. BMI: body mass index.

Table 2. Spearman Correlation Analysis of Lowest $\mathrm{SpO}_{2}$ and STOP-Bang Score, Age, and BMI

\begin{tabular}{lcccc}
\hline \multicolumn{1}{c}{ Items } & Coefficient & \multicolumn{1}{c}{$95 \%$ Cl for B } & SE & P value \\
\hline STOP-Bang score & -0.774 & -0.855 to -0.649 & 0.054 & 0.001 \\
Age & -0.201 & -0.418 to 0.032 & 0.117 & 0.137 \\
BMl & -0.288 & -0.518 to -0.013 & 0.142 & 0.031 \\
\hline
\end{tabular}

Cl: confidence interval, SE: standard error of coefficient B, BMl: body mass index.

Table 3. Odds Ratios of STOP-Bang Questionnaire Items

\begin{tabular}{lccc}
\hline \multicolumn{1}{c}{ Questionnaire } & Odds ratio & P value & $95 \% \mathrm{Cl}$ \\
\hline Snoring & 1.471 & $<0.001^{*}$ & 1.124 to 1.924 \\
Tired & 0.846 & 0.406 & 0.754 to 0.950 \\
Observed & 6.000 & $0.025^{*}$ & 1.086 to 33.145 \\
Pressure & 1.444 & $0.001^{*}$ & 1.118 to 1.866 \\
Age & 1.205 & 0.188 & 1.059 to 1.372 \\
Gender & 2.143 & 0.381 & 0.391 to 11.731 \\
\hline
\end{tabular}

Cl: confidence interval. *P value $<0.05$. 
Table 4. Patients Who Experienced Hypoxic Events $(n=8)$

\begin{tabular}{|c|c|c|c|c|c|c|}
\hline Case no. & Age (yr) & Gender & BMI $\left(\mathrm{kg} / \mathrm{m}^{2}\right)$ & STOP-Bang score & Risk & Lowest $\mathrm{SpO}_{2}(\%)$ \\
\hline 6 & 64 & $M$ & 22.1 & 5 & High & 81 \\
\hline 12 & 81 & $M$ & 24.5 & 5 & High & 88 \\
\hline 22 & 70 & $M$ & 27.2 & 5 & High & 88 \\
\hline 23 & 64 & $\mathrm{~F}$ & 22.3 & 4 & Intermediate & 86 \\
\hline 35 & 71 & $M$ & 25.0 & 5 & High & 88 \\
\hline 46 & 67 & $M$ & 30.1 & 4 & High & 88 \\
\hline 47 & 62 & $\mathrm{~F}$ & 22.8 & 5 & High & 85 \\
\hline 55 & 64 & $M$ & 29.7 & 4 & High & 84 \\
\hline
\end{tabular}

Values are expressed as numbers.

the incidence rate of OSA is high, as reported by Shin et al. [16]. OSA has a causal relationship with hypoxia during sedation, and severe hypoxia may be associated with increased mortality and morbidity [6]. Predicting those who are more likely to experience hypoxia will help us prepare for hypoxia during sedation.

The Berlin questionnaire, the STOP-Bang questionnaire, and the Epworth sleepiness scale are widely used as OSA screening tools. Among them, the STOP-Bang questionnaire is relatively accurate with high sensitivity and can be used for the early diagnosis of OSA, even in an environment where polysomnography is difficult to perform [17]. In addition, the STOP-Bang questionnaire uses only eight items to calculate the risk of OSA. The eight items include BMI, gender, age, neck circumference, hypertension under treatment, daily drowsiness, snoring, and apnea during sleep $[9,10]$. Therefore, the STOP-Bang score was selected as an index correlated with $\mathrm{SpO}_{2}$ in relation to hypoxia during sedation, since all items can be easily confirmed in advance with preoperative anesthesia evaluation without additional monitoring devices.

As a result, the STOP-Bang score and the lowest $\mathrm{SpO}_{2}$ showed a negative correlation (coefficient $=-0.774,95 \% \mathrm{CI}$ : -0.855 to $-0.649, \mathrm{SE}=0.054, \mathrm{P}<0.001)$. Although there were age and BMI items in the STOP-Bang questionnaire, weak interactions were observed between oxygen saturation and actual age or BMI. The reason for this low correlation is thought to be the binary classification of these items, such as age of 50 years or older and BMI above $35 \mathrm{~kg} / \mathrm{m}^{2}$.

Hypoxic events occurred in eight patients, hypoxia was resolved in two patients by supplying oxygen through a simple mask, but the other 6 patients resolved hypoxia through jaw thrust or manual ventilation. In 4 of these cases, the dexmedetomidine infusion rate was adjusted to half. This result is

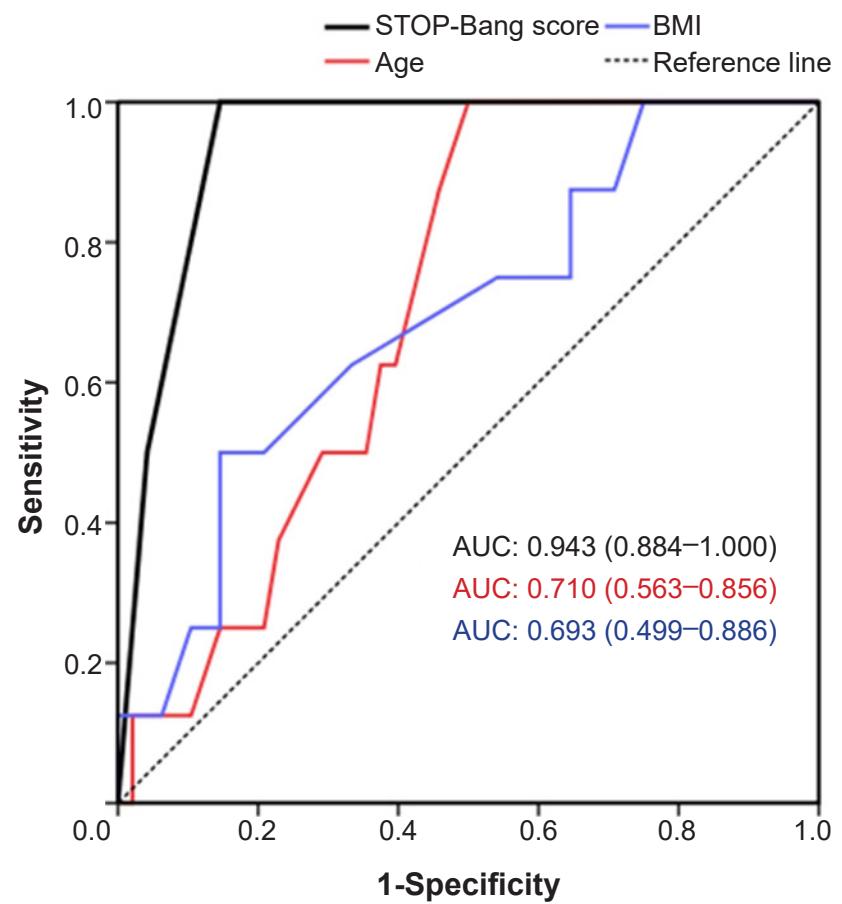

Fig. 3. Receiver operating characteristic curves and area under the curve (AUC) for STOP-Bang score, age, and body mass index (BMI).

thought to have been influenced by their relatively high ages. The mean age of the hypoxic event group (69.5 \pm 6.6 ) was higher than that of the group without events (59.8 \pm 15.3$)$.

ROC analysis was performed to check whether the STOPBang score is valid as a diagnostic tool for predicting hypoxia (Fig. 3). Consequently, the STOP-Bang score showed a stronger association with hypoxic events than the age and BMI of the patients. The cut-off value of the STOP-Bang score for predicting hypoxic events was 4 points. Previous studies by Chung et al. [13] showed a cut-off value of 3 points for the STOP-Bang score. This may be a result of the differences in the average BMI and the average neck circumference, proba- 
bly due to the nationality of the patient sample. The item " $\mathrm{B}$ " did not apply to any patient, and item "N" applied to only one patient. In this study, the cut-off value of the BMI was 27. According to the Asia-Pacific obesity diagnosis standards, obesity is defined as a BMI of $>25$, and high obesity is defined as a BMI of $>30$. It seems that the standard points for BMI and neck circumference for the STOP-Bang questionnaire are quite high for most Korean patients. This is considered the reason for the maximum STOP-Bang score of 5 in this study.

In the odds ratio analysis for 8 individual items, the "Snoring," "Observed," and "Pressure" items were statistically significant. In particular, the "Observed" item showed a very high odds ratio of 6.0, compared with those of the other two items, which were nearly 1.4. Although it usually refers to observation by non-medical personnel, this result is consistent with the intuition that the "Observed' item is highly correlated with OSA, and similar reports have been made in studies such as Neves Junior et al. [18].

The limitation of this study was that the oxygen mask with airway adjustments was immediately applied for patient safety when the $\mathrm{SpO}_{2}$ decreased to $92 \%$ and lasted more than 30 s or rapidly decreased to less than $90 \%$. Therefore, it was not possible to accurately evaluate the reduction in $\mathrm{SpO}_{2}$ in the high-risk group. In addition, since there is a part in which the patients answer the questions themselves, it is difficult to calculate the correct score if there is an incorrect answer from the patient, whether intended or not.

The duration for the lowest $\mathrm{SpO}_{2}$ was not directly collected, and the times of occurrence could not be analyzed. The 5-min interval recording system did not reflect the saturation in real-time, and it was impossible to collect retrospective data. However, according to the author's experience, most cases of hypoxia occurred nearly immediately after the loading dose was fully administered. There may be bias due to oxygen supply, and further research is needed.

In conclusion, we confirmed that the STOP-Bang was correlated with $\mathrm{SpO}_{2}$ in patients undergoing dexmedetomidine sedation in this study. This study proposes the use of the STOP-Bang score for preoperative evaluation and sedation management. The addition of the "observed" item for the pre-anesthesia evaluation may also be helpful.

\section{SUPPLEMENTARY MATERIALS}

Supplementary data including a STOP-Bang questionnaire can be found online at https://doi.org/10.17085/apm.21011.

\section{CONFLICTS OF INTEREST}

No potential conflict of interest relevant to this article was reported.

\section{DATA AVAILABILITY STATEMENT}

The datasets generated during and/or analyzed during the current study are available from the corresponding author on reasonable request.

\section{AUTHOR CONTRIBUTIONS}

Conceptualization: Yusom Shin. Data curation: Minsu Yun, Sungwon Ryu, Seo Han. Formal analysis: Minsu Yun, Sungwon Ryu. Methodology: Yusom Shin. Project administration: Jiwook Kim. Investigation: Seo Han. Software: Minsu Yun. Supervision: Jiwook Kim, Yusom Shin. Writing - original draft: Minsu Yun, Yusom Shin. Writing - review \& editing: Minsu Yun, Yusom Shin.

\section{ORCID}

Minsu Yun, https://orcid.org/0000-0001-8634-8136

Jiwook Kim, https://orcid.org/0000-0001-9944-2113

Sungwon Ryu, https://orcid.org/0000-0001-6450-197X

Seo Han, https://orcid.org/0000-0002-6095-0935

Yusom Shin, https://orcid.org/0000-0002-4829-0866

\section{REFERENCES}

1. Borgeat A, Aguirre J. Sedation and regional anesthesia. Curr Opin Anaesthesiol 2009; 22: 678-82.

2. Elkalla RS, El Mourad MB. Respiratory and hemodynamic effects of three different sedative regimens for drug induced sleep endoscopy in sleep apnea patients. A prospective randomized study. Minerva Anestesiol 2020; 86: 132-40.

3. Chang ET, Certal V, Song SA, Zaghi S, Carrasco-Llatas M, Torre $\mathrm{C}$, et al. Dexmedetomidine versus propofol during drug-induced sleep endoscopy and sedation: a systematic review. Sleep Breath 2017; 21: 727-35.

4. Xu JK, Ye JY, Cao X, Zhang YH, Yuan XM, Zhao CM. [Polysomnographic comparation between dexmedetomidine-induced sleep and natural sleep]. Zhonghua Er Bi Yan Hou Tou Jing Wai Ke Za Zhi 2019; 54: 405-9. Chinese.

5. Amornyotin S. Sedation-related complications in gastrointestinal endoscopy. World J Gastrointest Endosc 2013; 5: 527-33. 
6. Geng W, Jia D, Wang Y, Jin S, Ren Y, Liang D, et al. A prediction model for hypoxemia during routine sedation for gastrointestinal endoscopy. Clinics (Sao Paulo) 2018; 73: e513.

7. Coté GA, Hovis CE, Hovis RM, Waldbaum L, Early DS, Edmundowicz SA, et al. A screening instrument for sleep apnea predicts airway maneuvers in patients undergoing advanced endoscopic procedures. Clin Gastroenterol Hepatol 2010; 8: 6605.e1.

8. Mathangi K, Mathews J, Mathangi CD. Assessment of perioperative difficult airway among undiagnosed obstructive sleep apnoea patients undergoing elective surgery: a prospective cohort study. Indian J Anaesth 2018; 62: 538-44.

9. Larner A, B Z. Screening for obstructive sleep apnoea using the STOPBANG questionnaire. Clin Med (Lond) 2018; 18: 108-9.

10. Pearson F, Batterham AM, Cope S. The STOP-Bang Questionnaire as a screening tool for obstructive sleep apnea in pregnancy. J Clin Sleep Med 2019; 15: 705-10.

11. Nagappa M, Liao P, Wong J, Auckley D, Ramachandran SK, Memtsoudis S, et al. Validation of the STOP-Bang Questionnaire as a screening tool for obstructive sleep apnea among different populations: a systematic review and meta-analysis. PLoS One 2015; 10: e0143697.

12. Kim KT, Cho YW. Real-world STOPBANG: how useful is STOPBANG for sleep clinics? Sleep Breath 2019; 23: 1219-26.
13. Chung F, Abdullah HR, Liao P. STOP-Bang Questionnaire: a practical approach to screen for obstructive sleep apnea. Chest 2016; 149: 631-8.

14. Oshita H, Ito N, Senoo M, Funaishi K, Mitama Y, Okusaki K. The STOP-Bang test is useful for predicting the severity of obstructive sleep apnea. JMA J 2020; 3: 347-52.

15. Kang D, Lim C, Shim DJ, Kim H, Kim JW, Chung HJ, et al. The correlation of heart rate between natural sleep and dexmedetomidine sedation. Korean J Anesthesiol 2019; 72: 164-8.

16. Shin HJ, Kim EY, Hwang JW, Do SH, Na HS. Comparison of upper airway patency in patients with mild obstructive sleep apnea during dexmedetomidine or propofol sedation: a prospective, randomized, controlled trial. BMC Anesthesiol 2018; 18: 120.

17. Chiu HY, Chen PY, Chuang LP, Chen NH, Tu YK, Hsieh YJ, et al. Diagnostic accuracy of the Berlin questionnaire, STOP-BANG, STOP, and Epworth sleepiness scale in detecting obstructive sleep apnea: a bivariate meta-analysis. Sleep Med Rev 2017; 36: $57-70$.

18. Neves Junior JAS, Fernandes APA, Tardelli MA, Yamashita AM, Moura SMPGT, Tufik S, et al. Cutoff points in STOP-Bang questionnaire for obstructive sleep apnea. Arq Neuropsiquiatr 2020; 78: 561-9. 